\title{
Petrophysical models to describe the pressure dependence of acoustic wave propagation characteristics
}

\author{
Judit Somogyiné Molnár • A. Kiss · M. Dobróka
}

Received: 27 August 2014 / Accepted: 16 October 2014 / Published online: 31 December 2014

(C) Akadémiai Kiadó 2014

\begin{abstract}
Understanding the relationship between pressure and rock physical parameters, such as acoustic velocities, elastic moduli, porosity is essential for exploring and exploiting of natural reserves. In this study we introduce petrophysical models which describe the relationship between acoustic $\mathrm{P}, \mathrm{S}$ wave velocities as well as quality factors and pressure. The models are based on the idea that the pore volume of a rock is decreasing with increasing pressure. On the basis of the models the pressure dependent Lamé coefficients and loss angles were deduced. Laboratory measured acoustic $\mathrm{P}$ and $\mathrm{S}$ wave velocities and quality factors as a function of pressure were inverted to prove the applicability of the models and to obtain that of parameters. The quality checked joint inversion results showed that the calculated and measured data matched accurately and also proved that the suggested petrophysical models perform well in practice.
\end{abstract}

Keywords Rock pressure $\cdot \mathrm{P}$ and $\mathrm{S}$ wave velocities and quality factors · Lamé coefficients . Loss angles $\cdot$ Petrophysical models

\section{Introduction}

The knowledge of pressure dependence of rock physical parameters has a key role in the accurate interpretation of geophysical measurement data. Investigation of acoustic wave velocities and elastic properties has a great significance in seismic practice. Acoustic velocities are measured in the laboratory mostly by using the pulse transmission technique (Toksöz et al. 1979). The detection of transverse wave arrival is a greater challenge than that of the longitudinal one. The reason is that at small transducer-receiver distances the differentiation of $\mathrm{P}$ and $\mathrm{S}$ waves is difficult, however, if the distances are increased then also the attenuation

J. Somogyiné Molnár $(\varangle) \cdot$ A. Kiss · M. Dobróka

Department of Geophysics, University of Miskolc, Egyetemváros, Miskolc 3515, Hungary

e-mail: gfmj@uni-miskolc.hu

J. Somogyiné Molnár · M. Dobróka

MTA-ME Research Group of Geoengineering, Egyetemváros, Miskolc 3515, Hungary 
increases and the signal-to-noise ratio decreases. The velocities of acoustic waves propagating through different types of rocks under varying load (Wyllie et al. 1958; Stacey 1976; Sengun et al. 2011) and pore pressure (Nur and Simmons 1969; Yu et al. 1993; Darot and Reuschlé 2000; He and Schmitt 2006) are often investigated. It is observed that pressure has greater influence on velocities in the beginning phase of loading, later it lessens and the velocities tend to a limit value. Two main principles were published to explain this process. After (Birch 1960) consideration the reason of the velocity increase is the decreasing pore volume with increasing pressure. Walsh and Brace (1964) explain it with the closure of microcracks. Experiments demonstrate that beside other factors the type of pore fluid (Toksöz et al. 1979; Khazanehdari and McCann 2005), the grain size (Prasad and Meissner 1992; Prasad 2002) have influence on the scale of pressure dependence. A nonlinear relationship was proved by several empirical equations (Eberhart Phillips et al. 1989; Freund 1992; Jones 1995; Khaksar et al. 1999; Wepfer and Christensen 1991), however in these equations only the regression parameters are given, they do not provide the physical explanation of the process. In the followings petrophysical models are introduced which remedies this deficiency.

Beside the $\mathrm{P}$ and $\mathrm{S}$ wave velocities the pressure dependence of quality factors $\left(Q_{\alpha}, Q_{\beta}\right)$ or rather the attenuation (absorption coefficients) are often also investigated. To determine these parameters for example the resonance, ultrasonic pulse propagation, spectral ratio or low-frequency methods (Christensen and Wepfer 1989) are used. There are several models in the international literature to explain the attenuation of elastic waves, among others the nonlinear friction model, the Biot model (Biot 1956a, b), the viscoelastic model (Bland 1960) and the elastic dispersion model. The theories for the pressure dependence of velocities (Birch 1960; Walsh and Brace 1964) are suitable for the description of the relationship between quality factor and pressure. Experiments denote, that the quality factors behave similarly to the velocities, a rapid nonlinear increase occurs at the beginning of loading (Toksöz et al. 1979). The shale content, saturation and type of saturant, grain size influence the scale of pressure dependence (Khazanehdari and McCann 2005; Prasad and Meissner 1992; Prasad 2002; Domnesteanu et al. 2002). With the increasing pressure the pore volume decreases (or the microcracks close), the contacts between the grains become better and better thus the measurable absorption coefficient decreases and the quality factor increases.

Models are the simplified reality, where we keep the most important features and neglect the properties which do not or not substantially influence the examined process. During the development of the following rock physical models, the seismic/acoustic wave propagation phenomena is discussed with the application of the constant Q model, where the velocities and the quality factors - like phenomenological features - are rock stress dependent. With the assumption of the constant Q model after determining the velocities and quality factors at any pressure by inversion, the pressure dependent elastic parameters (for example the Lamé coefficients) and the loss angles can be deduced.

\section{Petrophysical models describing the pressure dependence of acoustic velocities}

As it was already mentioned, two basic ideas were published to explain the pressure dependence of acoustic wave velocities. Walsh and Brace (1964) connected it to the closure of microcracks, but the physical and mathematical description of the relationship remained unexplained. Dobróka and Somogyi Molnár (2012) developed a rock physical model for the stress dependency of longitudinal wave velocity based on the number of open microcracks. The basis of the model is that if we create a $\mathrm{d} \sigma$ stress increase in the rock, we find that $\mathrm{d} N$ (the change of the number of open microcracks) is directly proportional to the applied $\mathrm{d} \sigma$ 
stress increase. At the same time $\mathrm{d} N$ is directly proportional to $N$. These assumptions are merged in the differential equation ( $\lambda_{N}$ is a material dependent rock physical parameter)

$$
\mathrm{d} N=-\lambda_{N} N \mathrm{~d} \sigma .
$$

Eq. (1) can be written in general form as

$$
\mathrm{d} X=-\lambda_{x} X \mathrm{~d} \sigma,
$$

where $X$ is an extensive quantity (e.g. number of open microcracks, pore volume or area) which significantly influences the pressure effect.

Choosing pore volume as external quantity and applying similar assumptions, a petrophysical model can be derived for Birch (1960) theory. Namely, that the decreasing pore volume is the reason for the increasing velocities under loading. Since both the longitudinal and transverse waves have an important role in the seismic exploration of geological structures, the extension of velocity model for $\mathrm{S}$ wave is reasonable. The model is based on the pore volume or rather the change in pore volume, which is phenomenological isotropic, hence the model for longitudinal wave velocity can be rewritten for the transverse wave by substituting the relating velocities. It is especially important because both phase velocities are required to give the pressure dependence of elastic moduli.

Let us introduce the parameter $V$ as the pore volume (in a unit volume of a rock). We assume that a $\mathrm{d} \sigma$ stress increase applied to the rock will generate a $\mathrm{d} V$ change in pore volume directly proportional to the change in stress. Eq. (2) summarize these assumptions in a differential equation and its solution

$$
\mathrm{d} V=-\lambda_{V} V \mathrm{~d} \sigma \rightarrow V=V_{0} \exp \left(-\lambda_{V} \sigma\right)
$$

where $\lambda_{V}$ proportionality factor is a material dependent rock physical parameter, $V_{0}$ is the pore volume at stress-free state $(\sigma=0)$. The negative signs represent that the increasing stress decreases the pore volume. As the volume does not show anisotropy, Eq. (2) are the base of the model equations for both the $\mathrm{P}$ and $\mathrm{S}$ waves. We assume also a linear relationship between the infinitesimal change of wave velocities ( $\mathrm{d} \alpha$ for $\mathrm{P}$ wave and $\mathrm{d} \beta$ for $\mathrm{S}$ wave) and the change in pore volume

$$
\mathrm{d} \alpha=-\kappa_{\alpha} \mathrm{d} V, \mathrm{~d} \beta=-\kappa_{\beta} \mathrm{d} V,
$$

where $\kappa_{\alpha}$ and $\kappa_{\beta}$ are proportionality factors, new material characteristics respectively for $\mathrm{P}$ and $\mathrm{S}$ waves. The negative signs represent that the velocity is increasing with decreasing pore volume. Combining the assumptions of Eqs. (2-3) and solve the differential equation one can obtain

$$
\begin{aligned}
& \mathrm{d} \alpha=\kappa_{\alpha} \lambda_{V} V_{0} \exp \left(-\lambda_{V} \sigma\right) \mathrm{d} \sigma \rightarrow \alpha=K_{1}-\kappa_{\alpha} V_{0} \exp \left(-\lambda_{V} \sigma\right), \\
& \mathrm{d} \beta=\kappa_{\beta} \lambda_{V} V_{0} \exp \left(-\lambda_{V} \sigma\right) \mathrm{d} \sigma \rightarrow \beta=K_{2}-\kappa_{\beta} V_{0} \exp \left(-\lambda_{V} \sigma\right),
\end{aligned}
$$

where $K_{1}$ and $K_{2}$ are integration constants which can be computed from Eq. (4) as $\alpha_{0}=$ $K_{1}-\kappa_{\alpha} V_{0}$ and $\beta_{0}=K_{2}-\kappa_{\beta} V_{0}$, where $\alpha_{0}$ and $\beta_{0}$ are the propagation velocities at stressfree state which can be measured in laboratory. In the framework of the model, the velocities of acoustic waves increase from $\alpha_{0}$ and $\beta_{0}$ (at zero pressure) to $\alpha_{\max }=\alpha_{0}+\Delta \alpha_{0}$ and $\beta_{\max }=\beta_{0}+\Delta \beta_{0}$ (at high pressure, when all the pores are closed). So, $\Delta \alpha_{0}$ and $\Delta \beta_{0}$ can be considered the velocity-drops (compared to the fully compacted state where the pore volume equals zero) caused by the presence of pores at zero pressure (Ji et al. 2007). With introducing the notations $\Delta \alpha_{0}=\kappa_{\alpha} V_{0}, \Delta \beta_{0}=\kappa_{\beta} V_{0}$ Eq. (4) can be rewritten in the forms

$$
\alpha=\alpha_{0}+\Delta \alpha_{0}\left(1-\exp \left(-\lambda_{V} \sigma\right)\right), \beta=\beta_{0}+\Delta \beta_{0}\left(1-\exp \left(-\lambda_{V} \sigma\right)\right)
$$


Equation (5) provide a theoretical connection between the propagation velocity and rock pressure. Note that in the range of high pressures, reaching a critical pressure (Anselmetti and Eberli 1997) the reversible range is exceeded and destruction of the sample may occur thus decreasing velocities can be observed. This effect is outside of our present investigations. Therefore these models are valid only in the reversible range. As it can be seen from the Eq. (5) the $\lambda_{V}$ is a common petrophysical parameter. The physical meaning of $\lambda_{V}$ can be given by introducing the notation $\Delta \alpha=\alpha_{\max }-\alpha$ and $\Delta \beta=\beta_{\max }-\beta$ (the velocity-drop caused by the presence of pores at pressure $\sigma$ ), Eq. (5) can be written in the forms

$$
\Delta \alpha=\Delta \alpha_{0} \exp \left(-\lambda_{V} \sigma\right), \Delta \beta=\Delta \beta_{0} \exp \left(-\lambda_{V} \sigma\right)
$$

Laboratory tests indicate that the various types of rock response in a different scale to pressure changes. This feature can be described with the sensitivity function, which is widely used in literature. Hence we introduce the (logarithmic) stress sensitivity of the velocity-drops as

$$
S(\sigma)=-\frac{1}{\Delta \beta} \frac{\mathrm{d} \Delta \beta}{\mathrm{d} \sigma}=-\frac{\mathrm{d} \ln (\Delta \beta)}{\mathrm{d} \sigma}=\lambda_{V} .
$$

By using Eq. (6) it can be seen that the petrophysical characteristic $\lambda_{V}$ is the logarithmic stress sensitivity of the velocity-drops (Dobróka and Somogyi Molnár 2012). Note that in the framework of our model this characteristic is the same for P and $\mathrm{S}$ waves.

\section{Rock physical models for the pressure dependence of quality factors}

The physical explanation of attenuation of elastic waves can be characterized in two ways (Toksöz and Johnston 1981). One type of the models explains the phenomenon of attenuation through generalized linear elastic equations (Hooke-law) or modified equations permitting some nonlinearity. The other part of models applies new physical and mathematical description to interpret possible attenuation mechanisms, which are related to the microscopic features of rocks and their behaviour during wave propagation. Following the latter policy a rock physical model was introduced for the pressure dependent quality factor of longitudinal wave by Dobróka and Somogyi Molnár (2012). Their model refers to the change in quality factors caused by the closure of microcracks. Similarly to the velocity models, the quality factor models describing the longitudinal and transverse wave attenuation can be derived for varying pore volume. Here the constant Q model is applied again.

The increasing stress causes compaction in the grain structure, e.g. the pore volume decreases. As a result increasing quality factors can be measured. Let us assume linear relationship between the change of pore volume $(\mathrm{d} V)$ and the change of quality factors $\left(\mathrm{d} Q_{\alpha}\right.$ and $\mathrm{d} Q_{\beta}$ ) and introduce Eq. (8) as model laws

$$
\mathrm{d} Q_{\alpha}=-\chi_{\alpha} \mathrm{d} V, \mathrm{~d} Q_{\beta}=-\chi_{\beta} \mathrm{d} V,
$$

where the $\alpha$ and $\beta$ indices represent the quality factors for P and S waves respectively, $\chi_{\alpha}$ and $\chi_{\beta}$ are proportionality factors and the negative signs represent that the decreasing pore volume results in increasing quality factor. Combining the Eqs. (2) and (8) the following relations can be written

$$
\mathrm{d} Q_{\alpha}=\chi_{\alpha} \lambda_{Q} V_{0} e^{-\lambda_{Q} \sigma} \mathrm{d} \sigma, \mathrm{d} Q_{\beta}=\chi_{\beta} \lambda_{Q} V_{0} e^{-\lambda_{Q} \sigma} \mathrm{d} \sigma .
$$

The quality factors at stress-free state $\left(Q_{\alpha 0}\right.$ and $\left.Q_{\beta 0}\right)$ can be measured, thus the integration constants can be calculated (similarly to the velocity models). Introducing the notations $\Delta Q_{\alpha 0}=\chi_{\alpha} V_{0}$ and $\Delta Q_{\beta 0}=\chi_{\beta} V_{0}$, Eq. (9) take the forms 


$$
Q_{\alpha}=Q_{\alpha 0}+\Delta Q_{\alpha 0}\left(1-e^{-\lambda_{Q} \sigma}\right), Q_{\beta}=Q_{\beta 0}+\Delta Q_{\beta 0}\left(1-e^{-\lambda_{Q} \sigma}\right),
$$

where $\lambda_{Q}$ is a common material dependent rock physical parameter. It can be seen from the model equations that the quality factors change also exponentially with the pressure. The $\Delta Q_{\alpha 0}$ and $\Delta Q_{\beta 0}$ mean the quality factor ranges for the $\mathrm{P}$ and $\mathrm{S}$ waves, the differences between the quality factors at the stress-free state and at maximal stress ( $\left.Q_{\alpha} \max , Q_{\beta} \max \right)$.

\section{The pressure dependence of Lamé coefficients and loss angles}

The most often applied model for describing the elastic properties of rocks is the model of linear elastic homogeneous isotropic body or Hooke-body. In this case the stresses arising in the medium depend linearly from the deformations and this relationship can be described with two elastic material characteristics, the Lamé coefficients ( $\mu$ and $\lambda$ )

$$
\mu(\sigma)=\rho(\beta(\sigma))^{2}, \lambda(\sigma)=\rho(\alpha(\sigma))^{2}-2(\mu(\sigma)),
$$

where $\rho$ is the density (regarded as constant), $\beta(\sigma)$ and $\alpha(\sigma)$ are the (pressure dependent) transverse and longitudinal velocities. By means of the rock physical models describing the pressure dependence of $\mathrm{P}$ and $\mathrm{S}$ wave velocities, the stress dependence of Lamé coefficients can be derived or rather model-like interpreted.

If measured data of quality factors are also available the pressure dependent moduli and dissipative parameters (loss angles $-\varepsilon, \varepsilon^{\prime}$ ) can be also deduced. With the assumption of constant Q model the Lamé coefficients are complexes

$$
\mu=\mu^{*}(1+i \varepsilon), \lambda=\lambda^{*}\left(1+i \varepsilon^{\prime}\right),
$$

where $\mu^{*}, \lambda^{*}$ are the real part of the Lamé coefficients, $\varepsilon, \varepsilon^{\prime}$ are the so-called loss angles for which

$$
\left.\operatorname{tg} \delta=\frac{\operatorname{Im}\{\mu\}}{\operatorname{Re}\{\mu\}}=\varepsilon, \operatorname{tg} \delta^{\prime}=\frac{\operatorname{Im}\{\lambda\}}{\operatorname{Re}\{\lambda\}}\right)=\varepsilon^{\prime} .
$$

(For small angles $\operatorname{tg} \delta \approx \delta$.) Solving the wave equations for body waves, the quality factors can be calculated as

$$
Q_{\beta}=\frac{1}{\varepsilon}, Q_{\alpha}=\frac{\lambda+2 \mu}{\lambda \varepsilon^{\prime}+2 \mu \varepsilon} .
$$

Although the velocities and quality factors are determined during the measurements, the "real" material characteristics are $\mu, \lambda, \varepsilon, \varepsilon^{\prime}$. The pressure dependent loss angles can be deduced as

$$
\varepsilon(\sigma)=\frac{1}{Q_{\beta}(\sigma)}, \varepsilon^{\prime}(\sigma)=\frac{\lambda(\sigma)+2 \mu(\sigma)}{\lambda(\sigma) Q_{\alpha}(\sigma)}-\frac{2 \mu(\sigma)}{\lambda(\sigma) Q_{\beta}(\sigma)} .
$$

To prove the applicability of the presented models, laboratory measured data published in literature were processed, finally the pressure dependent elastic (Lamé coefficients) and dissipative (loss angles) parameters were calculated.

\section{Samples}

Acoustic velocity data were obtained for Berea sandstone from Winkler and Murphy (1995) and for Conglomerate from He and Schmitt (2006). The chosen velocity and quality factor data of Coal Nr. 16 sample was measured by Yu et al. (1993). All authors applied the pulse 


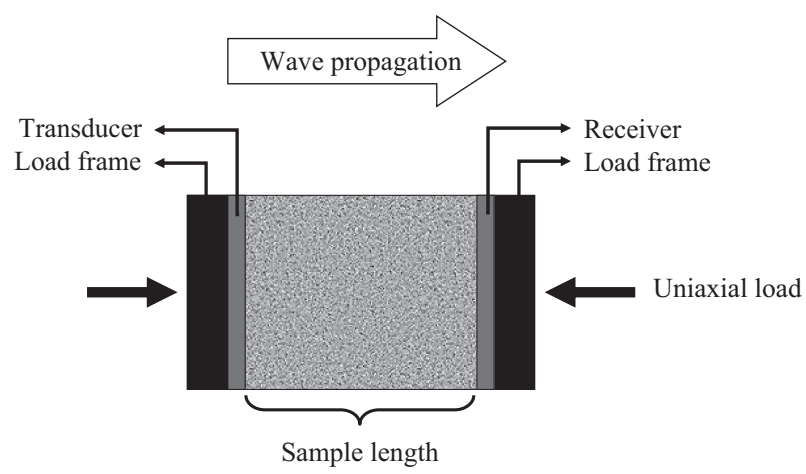

Fig. 1 Illustration of the pulse transmission technique

Table 1 Properties of the samples

\begin{tabular}{lccc}
\hline Sample & Characteristics & Other properties & Measured quantity \\
\hline $\begin{array}{l}\text { Berea sandstone } \\
\text { (Winkler and }\end{array}$ & $\begin{array}{c}\text { Homogeneous, } \\
\text { weakly cemented }\end{array}$ & $\begin{array}{c}\text { Composed of quartz } \\
\text { held together by }\end{array}$ & P and S wave velocities \\
medium-grained & silica, porosity & \\
& sandstone, shows & $16 \%$, permeability & \\
& microcracks & $75 \mathrm{mD}$, average bulk & \\
& & density $2,61 \mathrm{~g}^{3} \mathrm{~cm}^{3}$ & \\
Conglomerate (He & - & Bulk density & P and S wave velocities \\
and Schmitt 2006) & & 2,3 g/cm $\mathrm{cm}^{3}$, low & \\
& & porosity & \\
Coal Nr.16 (Yu et al. & Upper permian black & Originated from the & P, S wave velocities \\
1993) & coal, homogeneous, & Bulli Seam & and quality factors \\
& microbanded in the & & \\
& central locality & & \\
\hline
\end{tabular}

transmission technique to measure the $\mathrm{P}$ and $\mathrm{S}$ wave velocities and the spectral ratio technique (Toksöz et al. 1979) was used to determine quality factors. The former method means that $\mathrm{P}$ and $\mathrm{S}$ wave transducers and receivers are matched to the end caps of the samples and the travel time is measured (Fig. 1). The velocities can be calculated easily from the sample length and the travel time. In case of the latter method a reference specimen is used with very low attenuation properties and the same geometry as the tested specimen.

All measurements were carried out on dry cylindrical samples under uniaxial stress. The properties of the samples can be seen in Table 1 .

Note that at the measurements of Winkler and Murphy (1995) the compressional waves propagated parallel to the uniaxial stress and the transverse wave propagated perpendicular but with a polarization parallel to the uniaxial stress direction. The published measurement data indicate that the velocities and quality factors increase first strongly nonlinearly with increasing pressure (because the quantity of pores are relatively high in this region) then in the higher pressure range the increase in velocities (with increasing pressure) are moderate which can be attributed to the decrease of pore volume of rock sample, i.e. the pores are closing with pressure. 


\section{Case studies for the $P$ and $S$ wave velocity models}

Based on measurement data the petrophysical parameters $\left(\alpha_{0}, \Delta \alpha_{0}, \beta_{0}, \Delta \beta_{0}, \lambda_{V}\right)$ appearing in the model equations were determined by means of quality checked joint inversion method. In a joint inversion procedure we integrate all of the measurement data into one combined data vector and we give an estimate for the $\mathrm{P}$ and $\mathrm{S}$ wave velocity data in a single inversion algorithm, where $\lambda_{V}$ is a common petrophysical parameter connecting the two data sets. Equation (5) serve as forward modelling equations (model response functions) in handling the least squares-based joint inversion problem. The inversion results for both samples can be seen in Table 2 .

The estimation errors of the model parameters - which are in parenthesis after each parameter-were calculated using the formula given by Menke (1984)

$$
\sigma_{m_{i}}=\sqrt{\operatorname{cov}(\mathbf{m})_{i i}}
$$

which implies the elements of the main diagonal of the covariance matrix in parameter space ( $i=1, \ldots, 5$ in the given problem).

To confirm that $\lambda_{V}$ is a common petrophysical parameter we processed $\mathrm{P}$ and $\mathrm{S}$ wave velocity data sets by independent inversion method also. Table 3 contains the estimated values. By comparing them it can be seen that $\lambda_{V}$ determined by joint inversion falls between the ones calculated by independent inversion and they are approximately the same. Therefore it was reasonable to assume in the petrophysical model that $\lambda_{V}$ is the same for $\mathrm{P}$ and $\mathrm{S}$ waves.

With the estimated parameters, the velocities can be calculated at any pressure by substituting them into the model equations thereby applying Eq. (11) the pressure dependence of Lamé coefficients can be calculated. In case of the measured Lamé coefficients the $\mu$ and $\lambda$ values were calculated from the measured velocities. The inversion results are shown in Figs. 2, 3, where the solid lines show the calculated functions after 20 iteration steps while symbols represent the measured data.

The figures show that the calculated curves fit well to the measured data which proves that the petrophysical models describing the pressure dependence of $\mathrm{P}$ and $\mathrm{S}$ wave velocities can be applied well in practice.

For the characterization of the accuracy of inversion estimates the RMS $(D)$ value was calculated according to the following formula (Dobróka et al. 1991)

$$
D=\sqrt{\frac{1}{N} \sum_{k=1}^{N}\left(\frac{d_{k}^{(m)}-d_{k}^{(c)}}{d_{k}^{(c)}}\right)^{2}} \cdot 100[\%]
$$

where $\mathrm{d}_{k}^{(m)}$ is the measured data at the $k$-th pressure and $\mathrm{d}_{k}^{(c)}$ is the $k$-th calculated data which can be computed by the model equations. To characterize the reliability of the suggested petrophysical model the mean spread was also calculated by

$$
\mathrm{S}=\sqrt{\frac{1}{\mathrm{M}(\mathrm{M}-1)} \sum_{\mathrm{i}=1}^{M} \sum_{j=1}^{M}\left(\operatorname{corr}(m)_{\mathrm{ij}}-\delta_{\mathrm{ij}}\right)^{2}},
$$

where $\delta$ is a Kronecker-delta symbol (which equals 1 if $i=j$, otherwise 0 ), $M$ is the number of model parameters and $\operatorname{corr}(m)$ is the correlation matrix in parameter space, which provides the strength of linear relationships between each pair of model parameters. 


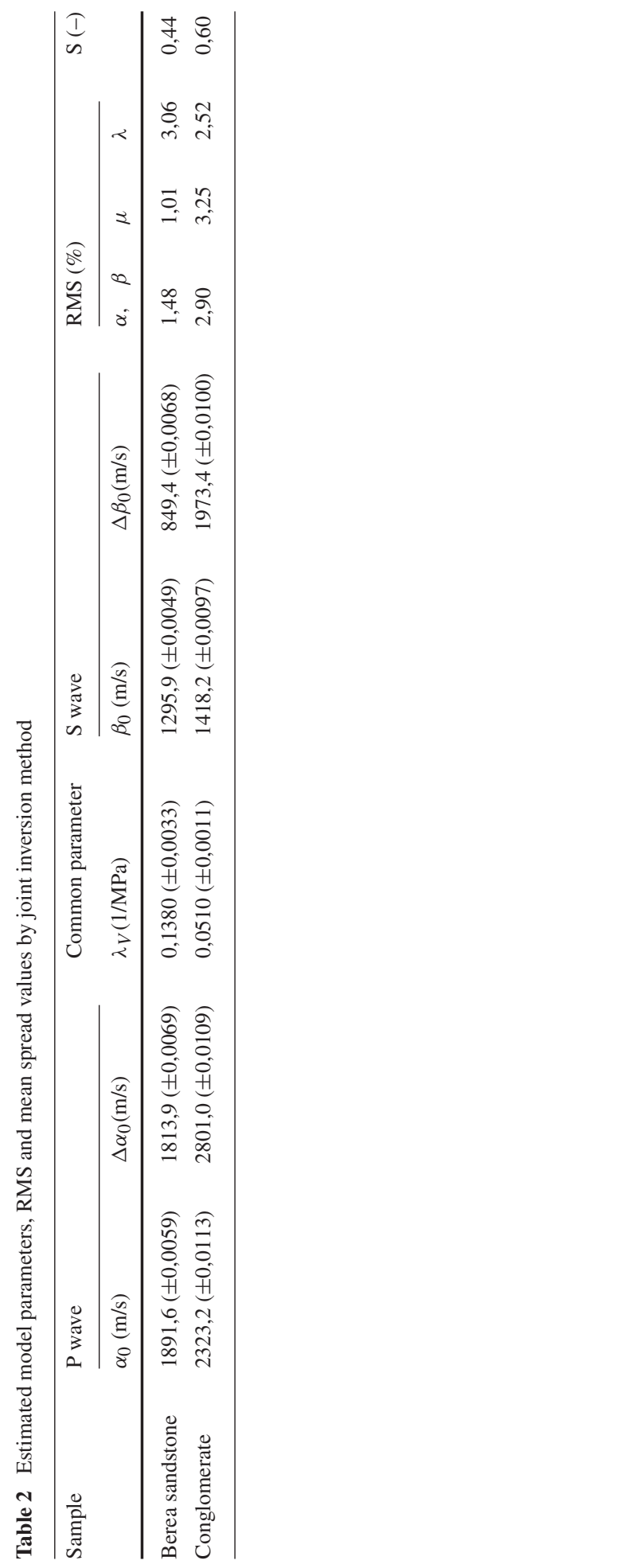


Table 3 Estimated $\lambda_{V}$ parameter by independent and joint inversion methods

\begin{tabular}{llll}
\hline Sample & $\begin{array}{l}\lambda_{V} \text { by independent } \\
\text { inversion }\end{array}$ & $\lambda_{V}$ by joint inversion \\
\cline { 2 - 3 } & P wave & S wave & \\
\hline Berea sandstone & 0,1470 & 0,1293 & 0,1380 \\
Conglomerate & 0,0504 & 0,0515 & 0,0510 \\
\hline
\end{tabular}
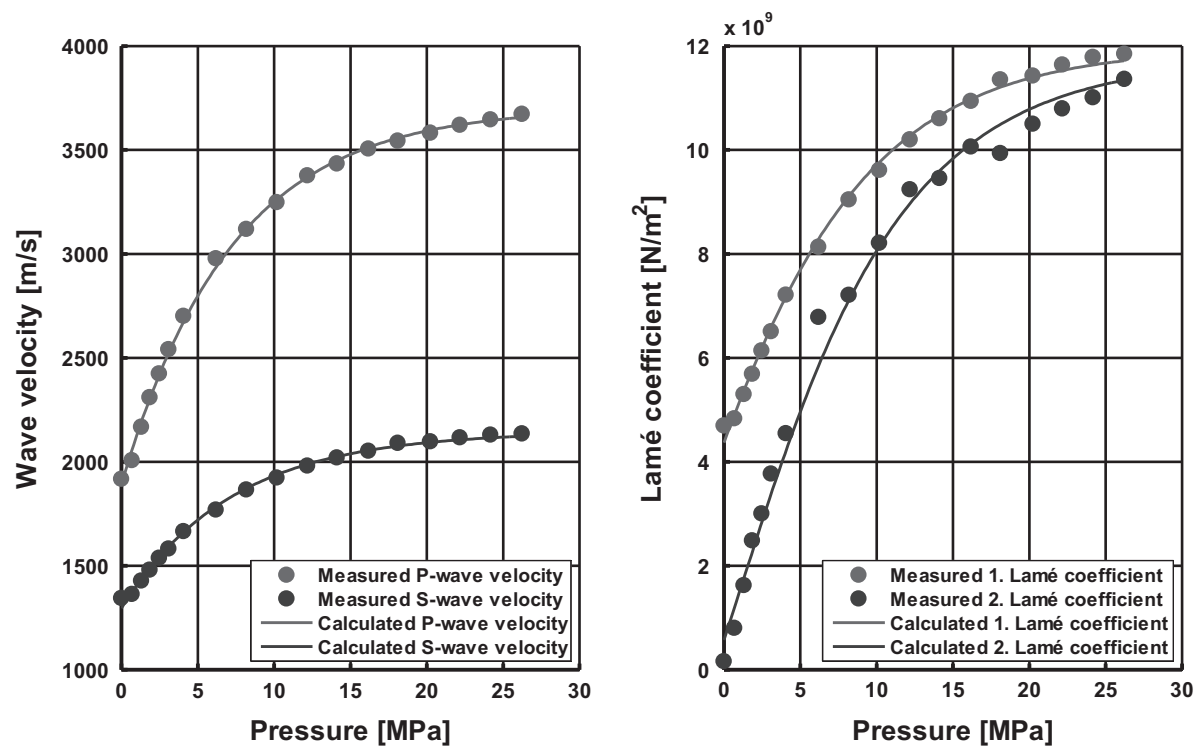

Fig. 2 Velocities/Lamé coefficients versus uniaxial pressure of Sample Berea sandstone. Data obtained by Winkler and Murphy (1995)

Table 2 contains also the calculated RMS and mean spread values for each sample in the last iteration step. It can be seen that the data misfits (RMS) were small and the mean spread values indicate that the parameters are in low-moderate correlation, so the inversion results are reliable. The application of the suggested models resulted in approximately the same data misfit on several sandstone samples. These results confirm the accuracy of the inversion estimates and the feasibility of the developed petrophysical models.

\section{Case study for the velocity and quality factor models}

Similarly to the previous section P, S wave velocity and quality factor data sets measured on the presented Coal Nr.16 sample was inverted by means of joint inversion processing. The inverse problem was significantly overdetermined; hence the inversion procedure was numerically stable and could be handled by a linear inversion technique. The calculated parameters together with their estimation errors can be seen in Table 4.

With the estimated parameters the velocities and quality factors can be determined at any pressure by means of the developed model equations. Figure 4 represents the results, the calculated Lamé coefficients and loss angles are produced by Eqs. (11) and (15). 

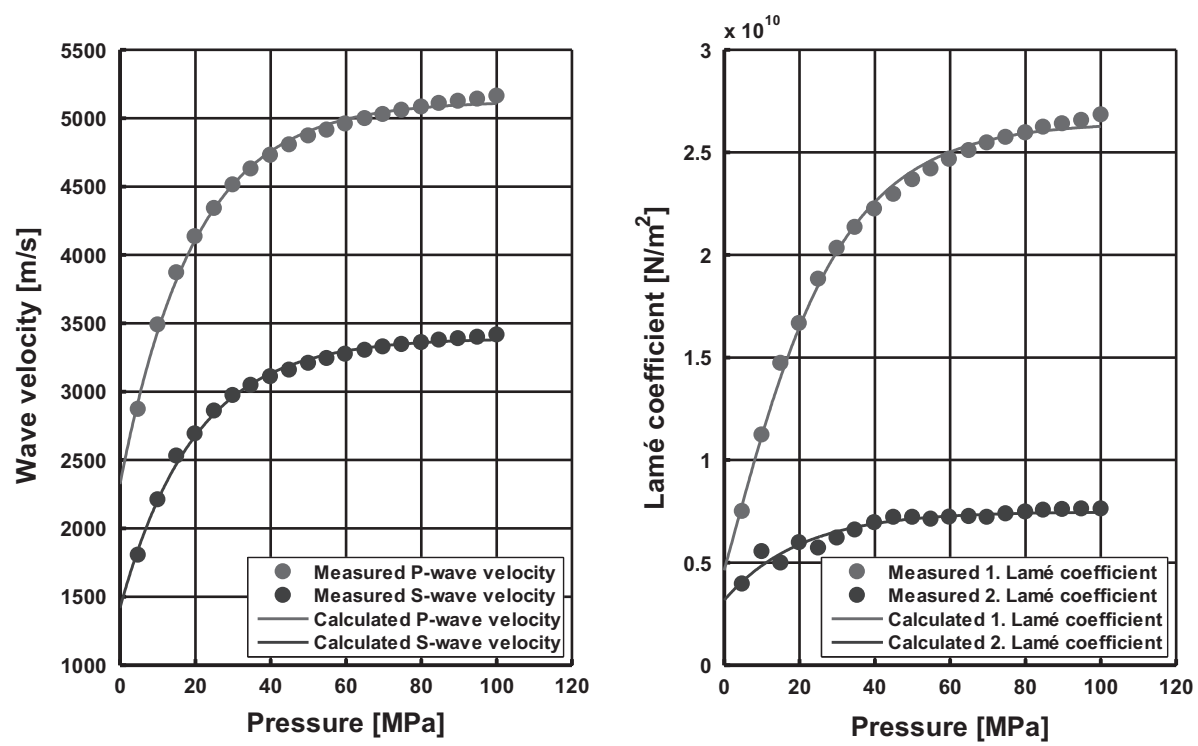

Fig. 3 Velocities/Lamé coefficients versus uniaxial pressure of Sample Conglomerate. Data obtained by He and Schmitt (2006)

As it can be seen the quality factors similarly to the seismic velocities increase with increasing pressure. The rate of increase is high at low pressures and levels off at higher pressures. The calculated curves are in good accordance with the measured data, which is strengthened by the calculated low RMS values (Table 4). In case of quality factors RMS values are higher than those at the velocities which can be explained by the difficulty of quality factor measurements. Even so the noise in data space is small-scale, which confirms the accuracy of the inversion results and the feasibility of the suggested petrophysical models for the explanation of the exponential relationship between the $\mathrm{P}$ and $\mathrm{S}$ wave velocities/quality factors and rock pressure. The moderate $(S=0,48)$ mean spread value confirms also that the inversion results are reliable.

\section{Conclusions}

We suggested petrophysical models for describing the connection between the velocity/quality factor of P, S waves and rock pressure. Exponential functions for an analytical description of the nonlinear velocity/quality factor versus pressure relationship are commonly used. The proposed models - in which six new petrophysical parameters $\Delta \alpha_{0}, \Delta \beta_{0}$, $\lambda_{V}, \Delta Q_{\alpha 0}, \Delta Q_{\beta 0}, \lambda_{Q}$ were introduced-provide physical meaning of the experimentally observed exponential pressure dependence. The models are valid only in the reversible range and are based on the idea that pore volume of the rock is decreasing with increasing pressure. For the longitudinal velocity and quality factor it states $\alpha=\alpha_{0}+\Delta \alpha_{0}\left(1-\exp \left(-\lambda_{V} \sigma\right)\right)$ and $Q_{\alpha}=Q_{\alpha 0}+\Delta Q_{\alpha 0}\left(1-e^{-\lambda_{Q} \sigma}\right)$. The same can be written for the transverse wave: $\beta=\beta_{0}+\Delta \beta_{0}\left(1-\exp \left(-\lambda_{V} \sigma\right)\right)$ and $Q_{\beta}=Q_{\beta 0}+\Delta Q_{\beta 0}\left(1-e^{-\lambda_{Q} \sigma}\right)$. In the equations $\alpha_{0}, \beta_{0}, Q_{\alpha 0}$ and $Q_{\beta 0}$ are the velocities/quality factors at zero pressure, $\Delta \alpha_{0}, \Delta \beta_{0}, \Delta Q_{\alpha 0}$ and $\Delta Q_{\beta 0}$ are the velocity/quality factor drops caused by the presence of pores as well as 


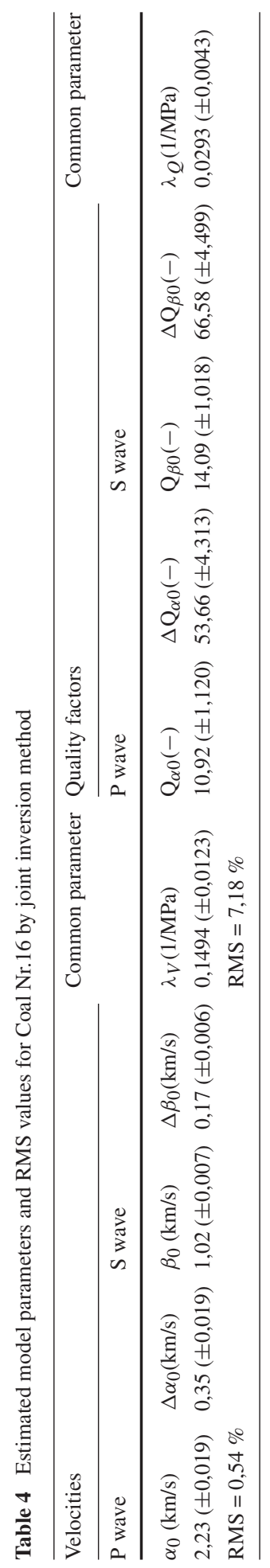



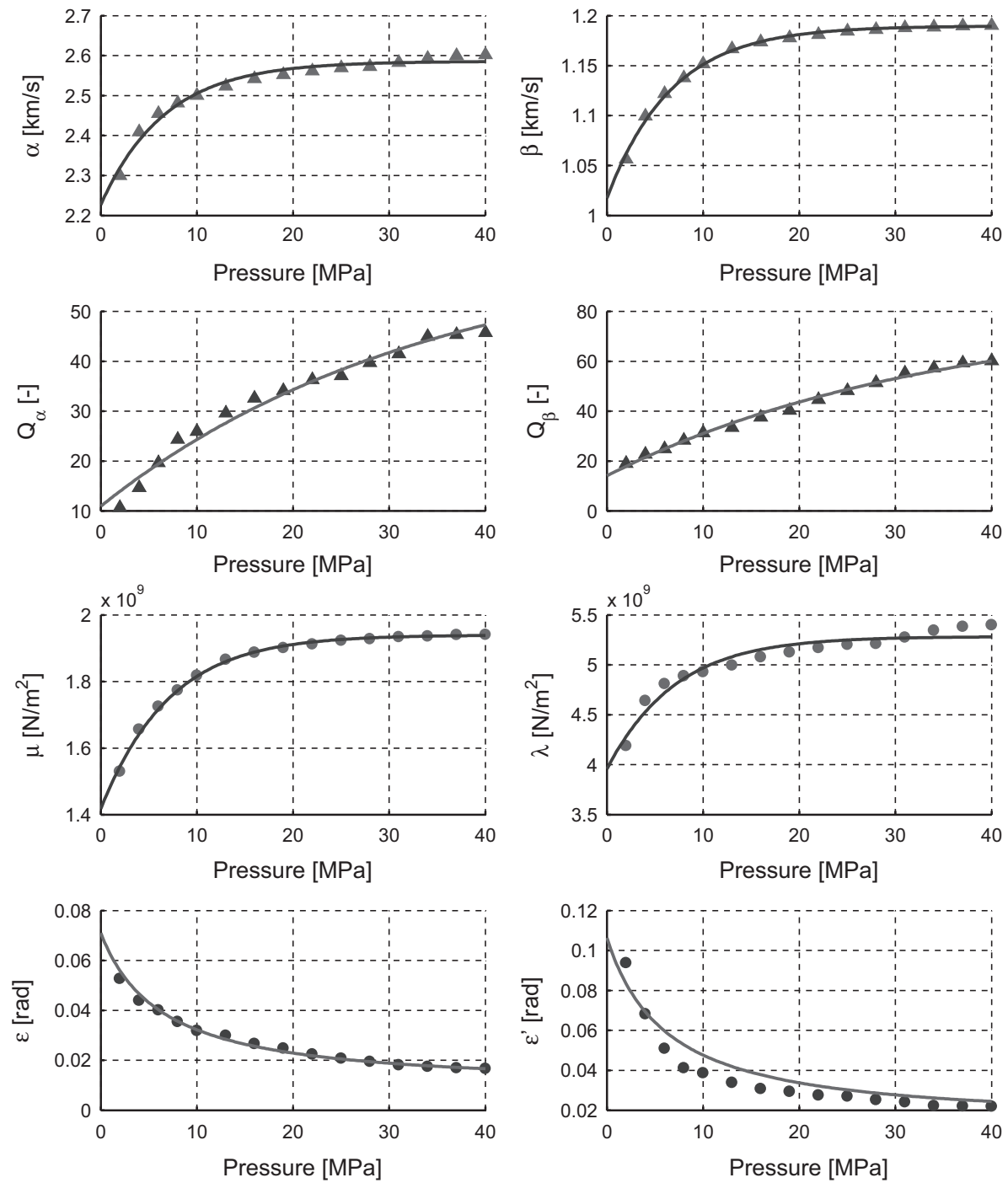

Fig. 4 Velocities/Lamé coefficients/loss angles versus uniaxial pressure of Sample Coal Nr.16 (solid linecalculated data produced by models, asterisks - measured data). Data obtained by Yu et al. (1993)

$\lambda_{V}, \lambda_{Q}$ are new petrophysical parameters. After estimating the mentioned model parameters by inversion procedure and calculating the velocities/quality factors, the pressure dependence of Lamé coefficients and loss angles can be deduced. To prove the applicability of our models it was tested on laboratory measured data published in literature and we found a very good agreement between measured and calculated data. Inversion results confirmed the accuracy and feasibility of the petrophysical models.

Acknowledgments The research of Judit Somogyi Molnár was supported by the European Union and the State of Hungary, co-financed by the European Social Fund in the framework of TÁMOP 4.2.4.A/2-11-12012-0001 'National Excellence Program'. The research of Anett Kiss and Mihály Dobróka was supported 
by the OTKA Project No. K 109441. The present investigations were based on the research work carried out in the framework of the Center of Excellence of Sustainable Resource Management.

\section{References}

Anselmetti FS, Eberli GP (1997) Sonic velocity in carbonate sediments and rock. In: Palaz I, Marfurt KJ (eds) Carbonate seismology. SEG, Tulsa, pp 53-74

Biot MA (1956a) Theory of propagation of elastic waves in a fluid-saturated porous solids, I: low frequency range. J Acoust Soc Am 28:168-178

Biot MA (1956b) Theory of propagation of elastic waves in a fluid-saturated porous solids, II: high frequency range. J Acoust Soc Am 28:179-191

Birch F (1960) The velocity of compression waves in rocks to 10 kilobars, Part 1. J Geophys Res 65:1083-1102

Bland DR (1960) The theory of linear viscoelasticity. Pergamon Press, Oxford

Christensen NI, Wepfer WW (1989) Laboratory techniques for determining seismic velocities and attenuations, with applications to the continental lithosphere. In: Pakiser LC, Mooney WD (eds) Geophysical framework of the Continental United States, vol 7. Geological Society of America, Memoir 172, Boulder, pp 91-102

Darot M, Reuschlé T (2000) Acoustic wave velocity and permeability evolution during pressure cycles on a thermally cracked granite. Int J Rock Mech Min Sci 37:1019-1026

Dobróka M, Somogyi Molnár J (2012) The pressure dependence of acoustic velocity and quality factor: new petrophysical models. Acta Geod Geophys Hung 47:149-160

Dobróka M, Gyulai Á, Ormos T, Csókás J, Dresen L (1991) Joint inversion of seismic and geoelectric data recorded in an underground coal-mine. Geophys Prosp 39:643-665

Domnesteanu P, McCann C, Sothcott J (2002) Velocity anisotropy and attenuation of shale in under- and overpressured conditions. Geophys Prosp 50:487-503

Eberhart-Phillips D, Han DH, Zoback MD (1989) Empirical relationships among seismic velocity, effective pressure, porosity, and clay content in sandstone. Geophysics 54:82-89

Freund D (1992) Ultrasonic compressional and shear velocities in dry clastic rocks as a function of porosity, clay content, and confining pressure. Geophys J Int 108:125-135

He T, Schmitt D R (2006) Velocity measurements of conglomerates and pressure sensitivity analysis of AVA response. In: Proceedings of $76^{\text {th }}$ SEG International Exposition and Annual Meeting, New Orleans, USA

Ji S, Wang Q, Marcotte D, Salisbury MH, Xu Z (2007) P wave velocities, anisotropy and hysteresis in ultrahigh-pressure metamorphic rocks as a function of confining pressure. J Geophys Res 112:B09204

Jones SM (1995) Velocities and quality factors of sedimentary rocks at low and high effective pressures. Geophys J Int 123:774-780

Khaksar A, Griffiths C, McCann C (1999) Effective stress coefficient for P- and S-wave velocity and quality factor in sandstone, Example from Cooper Basin, Australia. In: Proceedings of $69^{\text {th }}$ SEG Annual Meeting, Houston, USA

Khazanehdari J, McCann C (2005) Acoustic and petrophysical relationships in low-shale sandstone reservoir rocks. Geophys Prosp 53:447-461

Menke W (1984) Geophysical data analysis: discrete inverse theory. Academic Press, London

Nur A, Simmons G (1969) The effect of saturation on velocity in low porosity rocks. Earth Planet Sci Lett 7:183-193

Prasad M (2002) Acoustic measurements in unconsolidated sands at low effective pressure and overpressure detection. Geophysics 67:405-412

Prasad M, Meissner R (1992) Attenuation mechanisms in sands: laboratory versus theoretical (Biot) data. Geophysics 57:710-719

Sengun N, Altindag R, Demirdag S, Yavuz H (2011) P-wave velocity and Schmidt rebound hardness value of rocks under uniaxial compressional loading. Int J Rock Mech Min Sci 48:693-696

Stacey TR (1976) Seismic assessment of rock masses. In: Proceedings of Symp on Exploration for Rock Engineering, Johannesburg

Toksöz MN, Johnston DH (1981) Preface. In: SEG Geophysics reprint series No. 2 Seismic Wave Attenuation, $1-5$

Toksöz MN, Johnston DH, Timur A (1979) Attenuation of seismic waves in dry and saturated rocks: I laboratory measurements. Geophysics 44:681-690

Walsh JB, Brace WF (1964) A fracture criterion for brittle anisotropic rock. J Geophys Res 69:3449-3456

Wepfer WW, Christensen NI (1991) A seismic velocity-confining pressure relation, with applications. Int J Rock Mech Min Sci 28:451-456 
Winkler KW, Murphy WF (1995) Acoustic velocity and attenuation in porous rocks. In: Ahrens TJ (ed) Rock physics and phase relations: a handbook of physical constants. American Geophysical Union, Washington, D.C

Wyllie MRJ, Gregory AR, Gardner GHF (1958) An experimental investigation of factors affecting elastic wave velocities in porous media. Geophysics 23:459-493

Yu G, Vozoff K, Durney DW (1993) The influence of confining pressure and water saturation on dynamic elastic properties of some Permian coals. Geophysics 58:30-38 